\title{
O.S.P.
}

L'orientation scolaire et professionnelle

\section{Lallement, M. Temps, travail et modes de vie}

Paris : PUF

\section{Ginette Francequin}

\section{CpenEdition}

Journals

Édition électronique

URL : http://journals.openedition.org/osp/3356

DOI : 10.4000/osp.3356

ISSN : 2104-3795

Éditeur

Institut national d'étude du travail et d'orientation professionnelle (INETOP)

Édition imprimée

Date de publication : 1 septembre 2003

Pagination : 553-554

ISSN : 0249-6739

Référence électronique

Ginette Francequin, «Lallement, M. Temps, travail et modes de vie », L'orientation scolaire et professionnelle [En ligne], 32/3 | 2003, mis en ligne le 11 mai 2011, consulté le 23 octobre 2020. URL http://journals.openedition.org/osp/3356 ; DOI : https://doi.org/10.4000/osp.3356

Ce document a été généré automatiquement le 23 octobre 2020

(c) Tous droits réservés 


\section{Lallement, M. ${ }^{1}$ Temps, travail et modes de vie}

Paris : PUF

Ginette Francequin

\section{RÉFÉRENCE}

Paris : PUF

1 Le livre du sociologue Michel Lallement avec ses 210 pages denses, riches d'informations, bien écrites est un cadeau pour les praticiens des sciences humaines et sociales. Il l'est particulièrement pour les conseillers d'orientation-psychologues qui, pour informer sérieusement chaque élève ou étudiant sur les contextes des métiers et des emplois, ont besoin de connaître les transformations contemporaines du temps de travail. Cet ouvrage aide à se situer car il évalue les nouvelles politiques du temps de travail des dernières années avec un recul critique.

2 En effet, l'auteur rappelle que les historiens se sont penchés sur la transformation des cadres temporels de l'activité productive, montrant le poids des enjeux économiques. $\mathrm{Au}$ début du vingtième siècle, ils ont valorisé les actions tout à fait déterminantes des philanthropes et des militants du mouvement ouvrier. Si dans les années 80-90, le temps de travail est devenu un enjeu de compétitivité, les liens entre temps de travail et modes de vie n'ont pas encore été traités, car les interrogations ont surgi avec la mise en application des 35 heures. C'est sur cette question que Michel Lallement se penche avec le désir de prendre un recul historique et comparatif, d'interroger les implications sociales des politiques de flexibilité, de réfléchir à la place et au sens du travail au sein de nos sociétés.

3 Le matériau empirique donne de sérieuses fondations à ce livre, car il provient de quatre sources. Des entretiens semi-directifs ont été réalisés sur trois axes : parcours biographique, travail et temps de travail, changements hors travail. À ces enquêtes menées entre 1997 et 2002 par Michel Lallement, seul ou en collaboration avec des 
collègues, s'ajoutent des supports documentaires (littérature professionnelle et syndicale, écrits en circulation dans les organisations, tracts, sites Internet). La troisième technique mobilisée est l'observation de situations de travail et de logement des salariés; enfin la quatrième source est le traitement de données nationales et internationales.

Dans le premier chapitre, le décor historique est planté, grâce à une généalogie qui prend sa source avec la pointeuse, "ponctualité et régularité » sont exigées. Les ouvriers gagnent cinq dollars par jour, durant la semaine de travail de cinq jours, à partir de 1926 sous l'impulsion de Ford, sous condition d'une rationalisation des conduites dans et hors travail ${ }^{2}$.

5 Les enjeux de cette rationalisation peuvent être perçus dans le second chapitre grâce à la clarification de trois types d'articulations, celles des temps, au travail, du travail, de travail :

- Le temps au travail est illustré par des exemples sur l'intensification du travail, le contrôle accru des tâches et l'accroissement de l'autonomie qui vont de pair.

- Le temps du travail voit sa chronologie classique bouleversée au profit du travail qui, tout au long de la vie, « s'enchâsse différemment dans le reste de nos activités » avec ses contrats à durée déterminée, de l'intérim, des contrats aidés, des apprentissages qui s'allongent, pour arriver à ce que $10 \%$ de la population active soit concernée par ces diversifications. Les jeunes, les femmes et les populations peu qualifiées sont les premières victimes.

- Le temps de travail renvoie au décompte de la quantité de temps consacrée au travail, aux normes et pratiques puisque l'espérance de vie s'allonge. Si, sous Napoléon, le temps consacré au travail était de $70 \%$, un siècle plus tard, le chiffre se réduit de moitié. Aujourd'hui, avec les trente-cinq heures, nous sommes au travail durant moins de $20 \% \mathrm{du}$ temps de la vie éveillée...

6 Cette première partie du livre fourmille d'exemples qui rafraîchissent notre mémoire sur l'histoire du travail, nous propulsent dans la démarche sociologique avec ses emprunts explicites aux "grammaires durkheimienne et wébérienne", soit pour questionner le travail en tant qu'institution, soit pour privilégier la démarche comparative.

7 Dans la seconde partie, intitulée « les Mondes vécus de la flexibilité », quatre chapitres centrent l'analyse sur «Le temps des cadres, La recomposition des temps intégration par le travail ", "Le travail de nuit", "Le travail à temps partiel ». Là aussi, des situations exemplaires, significatives montrent combien l'intégration sociale des individus engage des interactions : elle se fait par une uniformisation des passions, et l'adoption de valeurs similaires. L'intégration des cadres est vue à travers deux entreprises, l'une fondée en 1947, l'autre est une start up, poussée en 1997. Le chercheur démontre comment la rationalisation du temps de travail des cadres banalise leur statut, contamine le travail des autres salariés, crée des lignes de fracture multiples. Les entreprises choisies confirment les effets de génération dans les modes d'intégration par le travail, ainsi que la persistance de fortes divisions entre les genres.

8 Le pourquoi travailler la nuit est posé par le biais d'exemples connus tels le travail des femmes d'une réglementation à l'autre, et la nuit en milieu hospitalier. Le lecteur suit Ghyslain, il somnole avec un œil ouvert, car tout peut arriver la nuit quand il est de garde avec ses 21 malades, à caler, changer; il mate une angoisse perpétuelle. Nous entrons dans l'intimité de Marion, de Jeanne, de Patricia, d'un couple de travailleurs de nuit dont une fille est handicapée. Le lecteur peut alors se demander avec le chercheur 
si le travail de nuit n'est pas accepté pour vivre moins difficilement une vie de travail avec le retour aux études des salariés ou pour aménager certaines contraintes familiales.

9 En ce qui concerne le travail à temps partiel, "palme d'or de la métamorphose du travail et des temps sociaux » depuis les années 80 , jamais vraiment choisi, jamais tout à fait contraint, il signe de manière incontestable les différences de classification et de salaire, les trajectoires divergentes, la reconnaissance inégale au sein des collectifs de travail, le risque accru de précarité avec une segmentation sexuée du marché du travail. À la Poste, grande banque sociale, en restructuration interne depuis plusieurs années, deux grands bureaux ont retenu l'attention du chercheur. Les entretiens avec les postières, Marion, Carole, Marie-Paule, Géraldine et Jeanne sont très révélateurs des conditions de travail qui poussent d'abord au temps partiel et se cumulent avec les conditions familiales (problèmes des modes d'accueil de la petite enfance).

La troisième partie du livre présente en une cinquantaine de pages des remarques critiques sur les facteurs à prendre en compte pour faire des comparaisons entre les pays européens. C'est difficile, mais finalement le chercheur opte pour une comparaison France-Suède. Les différences religieuses entre ces deux pays, les différences d'impact de la famille et des impératifs économiques sont notées pour interpréter mieux toutes les transformations du travail dont nous ne sommes qu'au stade des balbutiements, nous rappelle Michel Lallement.

11 Un livre intéressant, d'autant plus agréable qu'il se base sur de nombreuses données vivantes touchant aux questions très actuelles, fait une belle place au travail des femmes. L'auteur a aussi l'élégance de citer les travaux des chercheurs de son réseau, d'offrir une bibliographie nourrie, ce qui donne au lecteur des ouvertures, des curiosités.

12 En fermant l'ouvrage, l'envie m'a prise de me replonger dans les œuvres de Norbert Elias et de Michèle Perrot, d'ouvrir quelques revues, qui analysent les problèmes économiques et les actualités sociales françaises, de passer ainsi un moment avec les articles de Foster ou de Grossin. Ce livre donne le goût des autres, pour en savoir toujours plus!

\section{NOTES}

1. Professeur au CNAM et fondateur du GRIOT (Groupe de Recherches Interdisciplinaires sur l'Organisation du Travail. Laboratoire CNAM).

2. Selon des normes morales ? Un « département sociologique », doté de 50 inspecteurs est créé pour vérifier que le mode de vie des ouvriers est conforme à la morale de Ford. 


\section{AUTEURS}

\section{GINETTE FRANCEQUIN}

Maître de conférences en psychologie, Chaire de psychologie du travail, Laboratoire GRIOT. 